\title{
El proceso de integración educativa desde la perspectiva de cinco jóvenes universitarios con discapacidad visual de Concepción
}

\author{
The Educational Integration Process from the Perspective of Five Young College \\ Students with Visual Disabilities from Concepción \\ O processo de integração educacional do ponto de vista de cinco jovens \\ universitários com deficiência visual do Concepción \\ Cecilia Avendaño Bravo, ${ }^{a}$ Jessica Díaz Arias. ${ }^{b}$

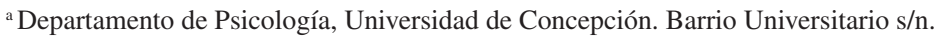 \\ Fono: 2204323. Correo electrónico: ceavendano@udec.cl

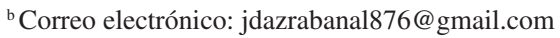

\begin{abstract}
RESUMEN
Este artículo presenta los resultados de un estudio de casos con enfoque biográfico-narrativo, cuyos objetivos fueron comprender la experiencia de jóvenes estudiantes universitarios discapacitados visuales sobre su proceso de integración en el sistema educativo, desde el período preescolar hasta la universidad, y analizar los factores que perciben como facilitadores y obstaculizadores. La muestra fue constituida por cinco jóvenes ciegos o con baja visión severa, de ambos sexos. La recolección de datos fue realizada mediante entrevistas en profundidad orientadas por un guion temático. Los resultados muestran que los padres y el centro de apoyo actúan como facilitadores mientras los profesores y pares en los establecimientos educacionales comunes son obstaculizadores en este proceso. Estos factores influyen en el desarrollo de capacidades psicológicas y relacionales fundamentales para la integración educativa y la inclusión social de estos jóvenes. A partir de la discusión de los resultados se sugieren algunas aplicaciones para mejorar este proceso.
\end{abstract}

Palabras clave: integración escolar, discapacidad visual, necesidades educativas especiales, igualdad de oportunidades.

\footnotetext{
ABSTRACT

This article presents the results of a case study, with biographical-narrative approach, which aimed to understand the experience of visually disabled university students and their integration process to the educational system - from kindergarten to university period - and analyze the factors perceived as facilitating and hindering. The sample consisted of five young people who were blind or with severe low vision, of both sexes. Data collection was conducted through in-depth interviews guided by a thematic script. The results show that parents and support center act as facilitators while teachers and peers in public educational institutions are understood as barriers in the process. These factors influence the development of psychological and relational capabilities which are fundamental in the educational integration and social inclusion of these youth. From the discussion of the results some methods to improve this process are suggested.
}

Key words: school integration, visual impairment, special educational needs, equal opportunities. 


\section{RESUMO}

Apresentam-se os resultados de um estudo de caso com abordagem biográfica-narrativa que objetivou compreender a experiência de estudantes universitários com deficiência visual em seus processos de integração no sistema de ensino, desde a creche à universidade e analisar os fatores percebidos como facilitadores e dificultadores. A amostra foi composta por cinco jovens cegos ou com baixa visão, de ambos os sexos. A coleta de dados foi realizada por meio de entrevistas em profundidade, orientadas por um roteiro temático. Resultados mostram que os pais e centro de suporte atuam como facilitadores, enquanto os professores e colegas em instituições públicas de ensino são obstaculizadores neste processo. Tais fatores influenciam o desenvolvimento das capacidades psicológicas e relacionais fundamentais para a integração educacional e de inclusão social desses jovens. A partir da discussão dos resultados, sugerem-se algumas aplicações para melhorar este processo.

Palavras chave: integração escolar, deficiência visual, necessidades educativas especiais, igualdade de oportunidades.

\section{INTRODUCCIÓN}

La integración escolar (IE) ha sido el enfoque predominante en la integración social de las personas con discapacidad en nuestro país. Este enfoque, basado en la normalización de la vida educativa de los alumnos con necesidades especiales (NEE), de quienes se espera que se integren al sistema educativo regular que, a su vez, debe realizar algunas adaptaciones curriculares y habilitar determinados apoyos, recursos y profesionales, exige a las personas con discapacidad su adaptación y asimilación a la oferta educativa (Valcarce, 2011). Así quedó establecido en el marco normativo y legal ${ }^{1}$ desarrollado desde principios de los 90', cuyas leyes han sido evaluadas recientemente como imprecisas, puesto que no detallan sus especificidades y mantienen ambigüedades en relación al tipo de educación propuesta (CLADE, CEJIL y Muñoz, 2009; Crosso, 2010).

De acuerdo con Gairín (2012), en Chile la atención a la diversidad en las políticas públicas referidas a la educación es un fenómeno que comienza recién en la segunda década del siglo XXI, con iniciativas que han tenido como actor clave al Estado, tanto por su rol normativo como financiero, y una perspectiva desde el principio de igualdad de oportunidades que no se ha traducido en instancias educativas suficientes para aquellos que tienen necesidades educativas especiales (entre otros grupos discriminados), en tanto que la promulgación de leyes anti-discriminatorias y las adecuaciones de la infraestructura escolar o el equipamiento pedagógico han ocurrido en forma lenta y poco eficaz. De hecho, sólo el año 2010, y en cumplimiento a las obligaciones que contrajo el Estado de Chile al ratificar la Convención de las Naciones Unidas sobre los Derechos de las Personas con Discapacidad y su Protocolo Facultativo, fue promulgada la Ley $N^{\circ} 20.422$ que se refiere a las normas sobre la igualdad de oportunidades e inclusión social de las personas con discapacidad (Fundación HINENI, 2002). Esta ley no ha sido aplicada en su totalidad y falta la dictación de algunos reglamentos importantes y del funcionamiento de todas las instituciones previstas, según señala un estudio preparado por el Departamento de Evaluación de la Ley de la Cámara de Diputados de noviembre de 2012.

\footnotetext{
El proceso de IE fue definido en el Decreto Supremo 490 del año 1990 (Ley N ${ }^{\circ}$.284) que estableció las bases legales para la integración social de personas discapacitadas y los Proyectos de Integración Educativa (PIE) como su principal herramienta y, posteriormente, en la Ley 20.370 del 2009, Ley General de Educación, que regula la educación en todos sus niveles y se refiere a los principios de equidad y calidad que deben estar presentes en el sistema educativo, marco donde se incluyen las necesidades educativas especiales (NEE). En la perspectiva de las NEE subyace el principio de que los fines de la educación deben ser los mismos para todos los alumnos, aunque el grado y el tipo de ayuda que necesiten sean distintos (ver Fundación HINENI, 2002).
} 
La integración en Chile tiene carácter voluntario, estrategia que según Blanco (2006) produce dos problemas: el carácter voluntario es adecuado para asegurar una actitud favorable de la comunidad escolar para acoger a los niños y jóvenes con discapacidad, pero tiene la desventaja de que otros establecimientos cercanos tienden a derivar a los alumnos con mayores dificultades a las escuelas de integración, de este modo, genera exclusión y discriminación. También restringe la oferta de integración a ciertos establecimientos, limitando la ampliación de la cobertura para un mayor número de niños y jóvenes con discapacidad y la libertad de los padres para elegir la escuela donde desean educar a sus hijos. Por otra parte, los proyectos de integración escolar (PIE) deben presentarse a la Administración Educativa (MINEDUC, 1990a), dependiendo de su aprobación la asignación de recursos humanos, materiales y financieros adicionales.

El Estado ha buscado materializar la integración utilizando la capacidad institucional pública (escuelas básicas y liceos), lo que ha llevado a realizar importantes cambios tanto en la escuela regular como en la educación especial (Tenorio, 2005). En general, se ha visto que las escuelas inician los procesos de IE por presión del medio o por decisión de sus directores o equipos técnicos, constituyéndose como una iniciativa de la autoridad educativa más que como una innovación de los centros educativos (Tenorio, 2005). Además, en muchos casos su elaboración es concebida como una actividad paralela dirigida al colectivo de los alumnos con discapacidad y asumida sólo por algunos docentes del establecimiento, en particular, por quienes tienen niños integrados en su aula (Marchesi, 2001).

En el año 2004, el MINEDUC designó una Comisión de Expertos de Educación Especial que diagnosticó un conjunto de factores que siguen siendo contradictorios y adversos para la atención a la diversidad, y que pueden sintetizarse en la idea de que existe una inconsistencia entre el diseño de las políticas y su implementación. También señaló dos barreras primordiales para la IE: a) las familias no tienen una participación real en el proceso educativo de sus hijos con PIE, y ocupan en éste un rol secundario, y b) las redes de apoyo al proceso de IE son casi inexistentes o no funcionan como debieran.

Para muchos actores del ámbito educativo, la integración constituye un desafío lejano de poder llevarse a la práctica, con costos de diversa índole, problemas asociados con la gestión organizacional y el desempeño profesional, lo que genera desmotivación en la escuela y una respuesta educativa inadecuada para los alumnos sujetos de integración, que continúan segregados y son llamados alumnos de integración (Tenorio y González, 2004).

Si bien algunos plantean que su inicio mediante experiencias aisladas a lo largo del país ha permitido demostrar que la integración es posible y beneficiosa para los niños y niñas y toda la comunidad educativa, teniendo un valor significativo en la promoción del derecho de las personas con discapacidad a educarse en contextos comunes (Consultora en Estudios, Asesoría y Planificación en Desarrollo Local, 2004), estudios recientes muestran que los profesores que trabajan en integración de alumnos con NEE no tienen asumida la atención a la diversidad como parte de su quehacer pedagógico, se sienten ajenos a este enfoque, expresan necesidad de capacitación y perfeccionamiento y de apoyo profesional externo, y poseen visiones negativas sobre los alumnos con NEE, basadas en estereotipos y prejuicios de los cuales no son conscientes y que se manifiestan en actitudes discriminatorias (Vega, 2009). $\mathrm{O}$ bien, tienen actitudes negativas como indiferencia, sobreprotección, bajas expectativas o positivas como la aceptación, perciben la IE con un enfoque clínico-rehabilitador y como una actividad compleja para la cual no tienen preparación ni experiencia (Damm, 2009). También se ha visto que en la mayoría de las mallas curriculares de formación de 
profesores los temas relativos a las NEE y su abordaje pedagógico no son esenciales ni forman competencias pedagógicas en el área (Tenorio, 2011).

En este artículo presentamos los resultados de un estudio que tuvo como objetivos principales comprender el proceso de IE desde la perspectiva de jóvenes estudiantes universitarios discapacitados visuales (DV), y analizar los factores que ellos perciben como facilitadores y obstaculizadores de este proceso. Un estudiante con NEE derivadas de la DV necesita recursos especiales y diferentes a los que la escuela proporciona habitualmente, lo que sólo será posible individualizando la enseñanza e intentando que cada cual alcance los objetivos de la educación de acuerdo con sus posibilidades (Blanco, 2006)². Sin embargo, a diferencia de otras discapacidades, en la enseñanza a estudiantes con DV no es necesaria la adaptación de los contenidos conceptuales, salvo en aquellos casos que presenten dificultades en el aprendizaje como consecuencia de la patología visual o limitaciones cognitivas asociadas con la DV. De este modo, la adaptación debe realizarse, sobre todo, en las estrategias metodológicas, las formas de evaluación y en los materiales de apoyo (Bermejo, Fajardo y Mellado, 2002).

\section{METODOLOGÍA}

El estudio fue realizado con metodología cualitativa. El diseño corresponde a un estudio de casos con un enfoque biográfico-narrativo.

\subsection{PARTICIPANTES}

La muestra fue seleccionada con el criterio de sujeto-tipo. Estuvo compuesta por cinco jóvenes estudiantes universitarios, 2 hombres y 3 mujeres entre 20 y 25 años de edad, solteros/ as, con discapacidad visual congénita ( 3 ciegos y 2 con baja visión severa), y que participaron de integración educativa en la provincia de Concepción. Ellos cursaban diversas carreras humanistas, 3 en la fase de inicio y 2 cercanos al término.

\subsection{ESTRATEGIA DE RECOLECCIÓN DE DATOS}

El método utilizado fue el de relatos autobiográficos, y tuvo el propósito de mostrar el testimonio subjetivo de los jóvenes sobre los acontecimientos vividos en el proceso de IE y sus valoraciones (Duero y Limón, 2008). La información fue obtenida mediante una entrevista en profundidad orientada por un guion temático con preguntas directrices. Este dispositivo aporta un material con la densidad y riqueza apropiada para los objetivos de este estudio (Sierra, 1998). Cada entrevista duró una hora y fue grabada en audio y,

\footnotetext{
La expresión NEE engloba tanto a los alumnos que requieren adecuaciones significativas, como a los que sólo requieren adecuaciones no significativas (Ainscow, 2001). Las orientaciones curriculares emanadas de la Reforma Educacional Chilena de 1990 contemplan tanto al alumnado que requiere una ayuda especial o extra para resolver sus dificultades de aprendizaje, así como la necesidad de establecer estrategias adecuadas para dar respuesta a las dificultades de cada alumno. Estas estrategias se denominan de forma genérica adaptaciones curriculares, y se distingue entre adaptaciones curriculares no significativas, que son aquellas que no alteran sustancialmente el currículo común, y adaptaciones significativas, las que pueden suponer la eliminación de contenidos esenciales de las diferentes áreas o asignaturas y la consiguiente modificación de los criterios de evaluación y las adaptaciones de acceso, dirigidas especialmente a los alumnos con discapacidades motoras, visuales y auditivas que originan dificultades de acceso (MINEDUC, 1990b).
} 
posteriormente, fue transcrita. Cuando en la transcripción de una entrevista se constató falta de información, se realizó una entrevista complementaria breve.

\subsection{ESTRATEGIA DE ANÁLISIS DE DATOS}

El tipo de análisis utilizado es el análisis de categoría (Gil, 1994), que fue orientado por los objetivos y la atención a categorías emergentes. Primero se realizó un análisis por categoría de cada entrevista. Luego, la información resultante fue incorporada en una matriz común, revisada varias veces atendiendo a que las categorías dieran cuenta del conjunto del material obtenido. A partir de la matriz definitiva, se hizo un análisis comparativo de las entrevistas por cada categoría. Para asegurar la calidad del análisis, éste fue realizado en paralelo por ambas investigadoras y discutido en cada una de sus fases.

\subsection{PROCEDIMIENTO}

Los participantes fueron contactados por una de las investigadoras. La participación fue voluntaria y con consentimiento informado. Debido a sus características, a los participantes se les explicó en forma oral los objetivos del estudio, en qué consistía su participación, el anonimato y confidencialidad de la información que entregaran, la posibilidad de negarse a responder o retirarse, y el uso exclusivo de la información para los fines de una investigación científica. Además, se solicitó su autorización para grabar la entrevista. Las entrevistas fueron realizadas de manera individual en dependencias del Departamento de Psicología de la Universidad de Concepción o en un lugar establecido de común acuerdo.

\section{RESULTADOS}

Los resultados del análisis de las entrevistas están organizados a partir de los factores externos presentes en el proceso de integración educativa y su rol como facilitadores $\mathrm{u}$ obstaculizadores del mismo, esto es, como contribuyentes o inhibidores del desarrollo de capacidades de los jóvenes. Las etapas iniciales de la IE hasta la enseñanza media están incorporadas en el análisis de los factores, mientras que la etapa universitaria es tratada en una sección aparte donde se abordan casi todos los factores anteriores más otros propios de este nivel de educación. Por último, se incluye un análisis evaluativo general del proceso de IE desde la perspectiva de los propios jóvenes.

\subsection{OBSTÁCULOS Y FACILITADORES EN EL PROCESO DE INTEGRACIÓN EDUCATIVA}

A lo largo del proceso de IE aparecen algunos factores externos del contexto inmediato que juegan un rol fundamental porque actúan como facilitadores u obstaculizadores del mismo, esto es, factores que desde la perspectiva de los jóvenes participantes tuvieron una presencia importante desde los inicios de su proceso de IE hasta llegar al nivel de estudios universitarios, constituyéndose en recursos de apoyo disponibles para ellos o cuya ausencia o la forma en que se manifestaron afectaron fuertemente sus vidas, ya sea con un impacto positivo o negativo. Los factores externos inmediatos predominantes como facilitadores del proceso de IE son los siguientes: los padres, otras personas significativas, las instituciones de 
apoyo y algunos aspectos técnicos vinculados con el proceso de enseñanza-aprendizaje. Por el contrario, las relaciones con sus pares y profesores en los establecimientos de educación común básica y media son los factores externos que principalmente se manifiestan en calidad de obstaculizadores del proceso, no así en la etapa universitaria.

Durante el proceso de IE, y facilitado por los factores externos inmediatos, surgen en los jóvenes recursos personales internos, de tipo psicológico, principalmente la capacidad de actuar con autonomía e independencia, y recursos relacionales, principalmente, habilidades sociales que son indispensables para una integración efectiva y exitosa.

\subsubsection{Los padres y el proceso de IE}

Los padres aparecen como precursores de la IE de sus hijos y constituyen el primer factor facilitador de este proceso. Este factor se refiere a una actitud proactiva general frente al hijo o hija con DV, siempre presente a lo largo de sus vidas. Consiste específicamente en no desfallecer en el difícil camino de búsqueda para conseguir ayuda temprana especializada para su hijo y, así, proporcionarle un futuro; también se dirige hacia la búsqueda de alternativas académicas y asegurarles apoyo especializado, jugando un papel determinante hasta la enseñanza media.

Los jóvenes relatan que sus padres vivieron una especie de duelo al enterarse de su condición de ceguera o DV, debiendo asumir con dolor una realidad que no esperaban. Luego del primer impacto, y en forma casi inmediata, iniciaron una compleja búsqueda de alternativas y orientaciones médicas que pudieran servirles para sacar adelante a sus hijos. En la época en que ellos nacieron, los padres carecían de orientación temprana respecto de especialistas, lugares o instituciones donde sus hijos pudieran recibir una atención profesional específica apropiada. En el ámbito médico existía un desconocimiento generalizado sobre el futuro de las personas ciegas y DV y, en algunos casos, la fase diagnóstica de la DV iba acompañada de desaciertos e imprecisiones.

En los relatos de los jóvenes, la resiliencia de sus padres es una constante a través de todo su proceso de habilitación. Por lo mismo, pese al escenario antes descrito, insistieron en la búsqueda de quien los orientara en la crianza y proyección de sus hijos hasta encontrar un centro especializado en la ciudad de Concepción, la Corporación de Ayuda al Limitado Visual (COALIVI), único por sus características entre los escasos centros de este tipo que entonces existían en el país. Los padres asumieron prontamente un rol activo en la gestión de los progresos de sus hijos.

Una vez que lograron integrarlos en un jardín infantil, y después de superar dificultades tales como lograr que los aceptaran en un jardín o encontrar uno que trabajara con niños con DV, en conjunto con el centro de apoyo iniciaron la búsqueda de establecimientos educativos comunes donde sus hijos pudieran continuar el proceso de IE de manera formal en la etapa educativa siguiente. En este proceso aprendieron especialmente qué implicaba la integración de las personas con DV, y pese a lo inusual y riesgosa que en aquellos años podía ser esta modalidad, todos estuvieron dispuestos a enviarlos a escuelas comunes. La actitud y convicción de los padres y de la institución de apoyo, que los llevó a apostar por esta opción educativa vanguardista, fue el motor que los impulsó en el proceso de lograr la IE de sus hijos. Además, a medida que sus hijos crecían, fueron inculcándoles que el lugar donde debían ir era la escuela, y que la meta era aprender al igual que sus hermanos, amigos o el resto de los niños. 
De las entrevistas se infiere que tanto los padres como el centro de apoyo preferían incorporarlos en establecimientos que contaran con ambos ciclos de educación, básica y media, con la finalidad de que los niños llevaran a cabo toda su educación formal en un único lugar. Es posible que también tuvieran el propósito de evitarles las dificultades adaptativas que podría acarrear un cambio, y de tener que iniciar ellos mismos otro largo y penoso peregrinaje. De hecho, los jóvenes que accedieron a establecimientos con estas características, permanecieron allí hasta terminar la enseñanza media. No todos los padres tuvieron la misma suerte. Aquellos que provenían de lugares alejados de la ciudad de Concepción, tuvieron más dificultades para encontrar establecimientos con ambos ciclos educativos que estuvieran dispuestos a recibir a sus hijos. Por otra parte, algunos padres buscaron también escuelas pertenecientes a alguna Iglesia acorde con sus valores, con la expectativa de que sus hijos con discapacidad fueran mejor acogidos y estuvieran más seguros. Sin embargo, esta premisa no siempre se cumplió en la realidad.

Una segunda constante que aparece en los relatos de los jóvenes es la sobreprotección de sus padres. En su afán de protegerlos, o debido a que sentían que su carga como estudiantes ciegos o con DV era demasiada alta, casi todos perciben que a veces sus padres limitaron o impidieron de alguna forma que ellos tuvieran espacios diferentes a los educativos, ya sea para desarrollar deportes o hobbies, salir con amigos o enfrentar los riesgos habituales en la vida de los niños, es decir, para llevar una vida normal. Así, la atención excesiva de los padres es vivida como un cierto obstáculo para su desarrollo.

$\mathrm{Al}$ parecer, también algunos padres presionaban a su hijo o hija con discapacidad para que aprovechara "cada" oportunidad que se le presentase y, más aún, los instaban a ir en busca de todas aquellas oportunidades que pudieran contribuir a su desarrollo. Esta presión provocaba en los jóvenes una especie de agotamiento emocional. Sentían que su tiempo estaba completamente ocupado con actividades que ni siquiera eran de su elección, que no disponían de tiempo para el ocio, o simplemente que no tenían la libertad de elegir no hacer nada. Algunos perciben que las demandas parentales constituyeron una sobreexigencia, que a veces obstaculizó su desarrollo en otras capacidades necesarias para la vida.

En general, los jóvenes perciben que su proceso de IE siempre implicó más esfuerzos y trabajo en comparación con las exigencias educativas de los demás niños. Desde la educación preescolar hasta cuarto medio, debieron asistir a diario tanto a la jornada de sus escuelas comunes como al centro de apoyo especializado en la jornada alterna. Lo anterior, tuvo como beneficio directo la transferencia inmediata de las técnicas instrumentales específicas relacionadas con la discapacidad, adquiridas en el centro de apoyo en su proceso de integración en las escuelas comunes. Asimismo, este ir y venir diario generaba un flujo de información constante para los especialistas del centro de apoyo sobre lo que ocurría con ellos en sus escuelas, pudiendo abordar prontamente la solución de los diversos problemas o requerimientos académicos y afectivos emergentes.

\subsubsection{Otras personas significativas}

Las otras personas significativas son aquellas que no siendo parte de la familia tuvieron un rol de apoyo importante para el niño con DV en el proceso de IE, que a veces mantienen hasta el presente. Los jóvenes señalan que estas personas les entregaron diversos tipos de apoyo, emocional, informacional e instrumental, siendo decisivas en sus logros. Aparecen mencionadas diversas personas que cumplen con esta condición: nanas, padrinos y, como 
Estudios Pedagógicos XL, N²: 45-64, 2014

EL PROCESO DE INTEGRACIÓN EDUCATIVA DESDE LA PERSPECTIVA DE CINCO JÓVENES UNIVERSITARIOS

CON DISCAPACIDAD VISUAL DE CONCEPCIÓN

se verá más adelante, en algunos casos es mencionado algún profesor o profesora de un establecimiento común, y la mayoría de las veces un/a profesor/a del centro de apoyo.

\subsubsection{Instituciones de apoyo para personas con DV}

Las instituciones de apoyo especializado también jugaron un rol fundamental en el desarrollo de los jóvenes. En particular, nos referimos a la Corporación de Ayuda al Limitado Visual, COALIVI, que en casi todos los casos partió entregando la primera orientación a los padres y la atención a ellos cuando eran bebés o durante sus primeros años de vida. Más adelante, cuando niños y adolescentes, les aportó técnicas instrumentales específicas relativas a su discapacidad, con el propósito de permitirles llevar una vida lo más normal posible e incorporarse y mantenerse en el sistema educativo común. La relación con la institución adquiere gran relevancia a través del tiempo, y la mayoría la siente como un espacio propio y una prolongación de su hogar.

El apoyo de COALIVI fue fundamental en su habilitación, según señalan los jóvenes, pero también en la orientación a sus padres y a la comunidad social inmediata (hermanos, abuelos, tíos, amigos). Ellos perciben que la orientación recibida fue realista en términos de las capacidades que tenían o podían desarrollar, y asertiva en relación con las limitaciones y dificultades que debían enfrentar. De alguna manera "les preparó el camino".

En sus relatos también aparecen los profesionales del centro de apoyo como personas que además de enseñarles técnicas específicas, los acogían a medida que iban creciendo y les ayudaban a obtener respuestas a las inquietudes y temores que surgían en su vida escolar. En general, valoran y agradecen el trabajo técnico pero también que los hicieron sentirse protegidos y queridos. Algunos agradecen, en particular, que sus profesores de apoyo asistieran al menos una vez a la semana a sus escuelas comunes.

\subsubsection{La relación con sus pares y profesores en establecimientos de educación común}

La postura y compromiso existente en el establecimiento sobre la IE y el manejo de las relaciones interpersonales en ese entorno resultan muy importantes. Las relaciones sociales durante todo el proceso de IE, tanto afectivas como de convivencia, son aspectos básicos para un resultado exitoso. En esta categoría surgen aspectos facilitadores y obstaculizadores que dependen de las condiciones creadas, y también de los propios individuos con DV, aunque en los relatos prevalecen las dificultades. Si bien algunos establecimientos educacionales, los menos, junto con aceptarlos en sus aulas se comprometieron con su IE tanto en el aspecto social como cognitivo, e intentaron cumplir con este compromiso durante toda su permanencia, otros olvidaron o desestimaron los requerimientos específicos de su condición de alumno ciego o con DV, mantuvieron posturas técnicas y pedagógicas poco claras y con efectos negativos, y los dejaron a la deriva en situaciones de conflicto en las relaciones sociales y afectivas con sus compañeros, a menudo, en situaciones con evidentes características de discriminación y violencia.

En general, los entrevistados consideran que el nivel de apertura de los profesores de la escuela común hacia el proceso de IE está sujeto a varios factores: de su grado de convicción con esta modalidad educativa, y que la IE es un principio, de la creencia de que es posible trabajar con la discapacidad con los recursos humanos y materiales disponibles y, más concretamente, de su voluntad para trabajar con niños y jóvenes discapacitados 
visuales. Así, en su paso por la educación básica y media hubo a veces profesores que trabajaron con ellos bajo la premisa de que primero eran alumnos y luego ciegos o DV, que les dieron confianza, los acogieron y creyeron en ellos y, sobre todo, tuvieron una buena disposición para trabajar en conjunto. Pero también encontraron un número importante de profesores con dificultades para hacerse cargo de alumnos con discapacidad en sus aulas.

Los jóvenes piensan que a los profesores que llevaban más tiempo en el sistema educativo les costaba más aceptar el hecho de tener alumnos con discapacidad en sus aulas, que el enfoque de la IE les causaba cierta desconfianza y lo vivían como algo impuesto. Tras estas actitudes y sentimientos creen que existía miedo, que se evidenciaba en el trabajo pedagógico y en las relaciones que establecían con ellos. Miedo al verse enfrentados con sus mitos sobre la ceguera, a no saber cómo actuar con ellos, a no tener respuestas, a no hacer lo correcto en su labor como profesor, en suma, miedo a lo desconocido. Desde su punto de vista, lo más lamentable es que tampoco mostraban iniciativa o intentaban conocer y aprender acerca de la DV.

También se encontraron con algunos profesores que los menoscabaron, los ignoraron dentro del aula o actuaron como si ellos no existieran, y que parecían negar sus posibles requerimientos especiales. A estos profesores parecía no interesarles la asesoría de la institución de apoyo y no consultaban sobre qué adaptaciones podían hacer en el caso del alumno con DV que tenían en el aula. Tampoco se daban tiempo para intentar trabajar con ellos, conversar, saber qué sentían, cuánto entendían o qué les resultaba más complicado. Desde la perspectiva de los jóvenes, esas actitudes y comportamientos denotaban una mala disposición o falta de interés para trabajar con ellos; simplemente, que su condición no les importaba o no era su problema. Por su parte, ellos sentían que intentar cambiar esa situación era muy difícil y omitían comunicarlo a sus padres o a otros profesores para no crear dificultades que finalmente podían repercutirles negativamente.

Finalmente, algunos profesores parecían no comprender qué es la IE, es decir, dar al alumno con NEE lo que necesita y no más que eso, y caían en el otro extremo. Manifestaban lástima por su condición, incurrían en una sobreprotección extrema haciendo diferencias notorias en relación al resto de los compañeros de curso para facilitarles la vida, sin entender que en lugar de ayudarlos los dañaban al restarle crédito a sus capacidades.

Por otro lado, en los relatos de las primeras relaciones con pares, sienten en general que en la etapa escolar comprendida entre primero y cuarto básico fueron aceptados, acogidos, incluso protegidos por ellos. La recuerdan como una etapa grata en que predominaron los afectos positivos. No obstante, a medida que pasaban los años y se iban convirtiendo en adolescentes, aparece una de las etapas socio-afectivas más difíciles de afrontar, marcada con rechazo y discriminación. Los jóvenes perciben que físicamente no cumplían ni con el patrón de belleza ni de popularidad valorados a esa edad. Además, en este periodo se cuestionaron y autorecriminaron por su condición de ciegos o DV, sobre todo por su apariencia, incluso con resentimiento por no ser igual al resto. La mayoría coincide en que el segundo ciclo básico, etapa comprendida entre quinto y octavo básico, es la etapa más conflictiva y difícil que les ha tocado enfrentar. Algunos asumen responsabilidades compartidas, de sí mismos por ser pasivos o poco tolerantes, y de los otros por su falta de empatía.

En el periodo en que comienza la enseñanza media, aunque existen episodios discriminatorios o de malos tratos de algunos compañeros, las relaciones comienzan a mejorar. La mayoría da cuenta de una apertura positiva de los demás hacia ellos, que les permite el desarrollo de relaciones de amistad que a veces permanecen hasta el presente. 
Estudios Pedagógicos XL, $\mathrm{N}^{\circ}$ 2: 45-64, 2014

EL PROCESO DE INTEGRACIÓN EDUCATIVA DESDE LA PERSPECTIVA DE CINCO JÓVENES UNIVERSITARIOS

CON DISCAPACIDAD VISUAL DE CONCEPCIÓN

No obstante, para algunos la relación con sus pares continuó siendo difícil, en ocasiones por la actitud de sus pares, y en otras por dificultades relacionales propias.

\subsubsection{Aspectos técnicos que facilitan la IE en los establecimientos comunes}

Éste es un factor que permite a los niños con necesidades educativas especiales (NEE) participar del currículo general del curso en que se encuentren y, básicamente, consiste en adecuaciones del currículo y en las distintas asignaturas en función de los requerimientos del alumno con discapacidad. Al respecto, los jóvenes dan a conocer que los aspectos técnicos que facilitaron su IE en los establecimientos comunes provenían de la acción o intervenciones del centro de apoyo, más que de la acción pedagógica de las escuelas.

En los relatos sobre el desarrollo de habilidades a través de técnicas instrumentales específicas y la valoración de su importancia para la DV, resulta relevante el desarrollo de ciertas capacidades por sobre otras. El valor atribuido depende de cómo estas capacidades inciden directamente en su nivel de independencia. En este sentido, queda de manifiesto que la independencia es fundamental para cualquier persona, pero que para las personas con DV es clave la capacidad de elegir y experimentar, de decidir sobre sus propias vidas, por tanto, les aporta las capacidades de autonomía y autodeterminación.

3.1.5.1. La importancia de la orientación y la movilidad (O y M) y de las actividades de la vida diaria (AVD) en el logro de la independencia física, psicológica y social

A través de un entrenamiento en técnicas específicas, con sus distintas etapas y niveles en directa relación con la madurez y el desarrollo, los niños con DV adquieren las herramientas necesarias para desplazarse en forma independiente. El uso del bastón, enmarcado en el proceso de $\mathrm{O}$ y $\mathrm{M}$, es un aspecto que los jóvenes señalan como primordial en sus vidas. Aprender a desplazarse con el bastón blanco, símbolo que mundialmente identifica a las personas con DV, provoca un vuelco en sus vidas. Les permite dejar de depender de otras personas para movilizarse y, así, aumenta su capacidad para ir hacia donde desean con mayor libertad.

De los relatos se puede concluir que aquellos jóvenes que son ciegos congénitos no tienen reparos en el uso del bastón. En cambio, para los jóvenes con visión deficiente o baja visión de alguna manera implicó asumir que su visión era insuficiente para permitirles un desplazamiento seguro, y asumen rezagos en su uso eficiente. Detrás de lo anterior es posible que en algunas ocasiones estén los padres, quienes a pesar de saber que sus hijos padecen una DV no siempre los tienen totalmente asumidos como personas ciegas, y donde el bastón pareciera que viene a recordarles que en un sentido estricto sí lo son. Sin embargo, todos señalan que con el tiempo asumen la importancia del uso del bastón en sus vidas como una herramienta que propicia la independencia deseada.

Los jóvenes también se refieren a las AVD como un área esencial para el logro de una independencia personal y social, les permite participar en distintos ámbitos sociales $\mathrm{y}$, además, incide directamente en la autoimagen y el autoconcepto. Implica el manejo en aspectos de la cotidianeidad, sean estos muy simples o más complejos, tales como comer usando cubiertos o cocinar sus alimentos, hasta viajar solos a otros países.

El abordaje de esta área en la institución de apoyo no está libre de críticas. Los jóvenes consideran que debiera tener un rol más protagónico, que debiera existir un mayor énfasis 
en el aprendizaje de estas técnicas que favorecen la independencia. Señalan que para ellos, como alumnos integrados, el trabajo en esta área no fue sistemático, quedando la mayoría de las veces relegado y por detrás de los requerimientos del área cognitiva. Asimismo, en cuanto a la orientación a los padres, creen que es importante enfatizar la relevancia de adquirir y poner en práctica las técnicas aprendidas, de manera que así como ellos tienen conciencia y recuerdan siempre a sus hijos con DV la importancia de estudiar, pudieran también recordarles la importancia que tendrá en sus vidas valerse por sí mismos.

\subsubsection{El desarrollo de habilidades sociales y cognitivas}

Las habilidades sociales y cognitivas son abordadas principalmente a través de un trabajo específico y guiado desde la institución de apoyo hacia las personas con DV. En general, los jóvenes coinciden en que ambos tipos de habilidades son fundamentales y deben desarrollarse al máximo, pero concluyen que en el caso de las personas con DV las habilidades sociales son las que finalmente priman en su vida de adultos. Éstas resultarían decisivas para integrarse plenamente en los ambientes sociales, educativos y recreativos, entre otros. Además, reiteran que en las áreas de apoyo del centro especializado en DV el énfasis estuvo puesto en el desarrollo de las habilidades cognitivas, para cubrir los requerimientos que la educación común exigía, mientras el desarrollo de las habilidades sociales ocupó un segundo plano, lo que finalmente les ha pasado la cuenta y ha dejado en evidencia sus desventajas en este sentido.

\subsection{LA INTEGRACIÓN A LA UNIVERSIDAD}

Los jóvenes tienen diversas aprehensiones en torno a su integración a la universidad. Antes de incorporarse, éstas se refieren a sus competencias y se expresan como dudas y temores acerca de si poseen las capacidades necesarias para la universidad, si podrán asumir exigencias mayores o establecer relaciones sociales con sus nuevos compañeros. Luego, en la fase inicial de integración universitaria, resurge el miedo a vivir situaciones de discriminación, del que alguna vez fueron víctimas. También los atemorizan experiencias y fracasos escolares anteriores, aunque antes hayan demostrado capacidad y hayan tenido un buen rendimiento, porque piensan que la universidad es distinta en todo ámbito de cosas. Y aunque evalúen que poseen capacidades, continúan dudando sobre su capacidad de enfrentarse con las nuevas exigencias académicas y sobre las formas de relacionarse con sus pares y profesorado, cómo manejarán mejor los tiempos o se desplazarán a lugares alejados y desconocidos incluso en los propios recintos universitarios. En general, una vez integrados efectivamente, parecen superar con relativa rapidez dichos temores y dudas.

Todos ellos optaron por carreras humanistas, principalmente, porque creen que en éstas tienen mayores posibilidades de realizar estudios con éxito, en parte porque durante su escolaridad obtuvieron más logros en las asignaturas humanistas, aunque no fueran sus favoritas, y también porque creen que su discapacidad implica limitaciones para las áreas científicas. Sin embargo, no cualquier carrera del área humanista resultaría apropiada per $s e$, dado que algunas carreras humanistas estarían mejor organizadas que otras. Para ellos es importante la organización en las materias y contenidos porque conocer de antemano los requerimientos y anticipar lo que necesitan hacer les permite planificar sus esfuerzos. Esta 
necesidad está asociada con las prácticas y características de cómo aprenden las personas con DV; organizarse, tener una rutina y trabajar con esquemas establecidos es muy común y efectivo en su aprendizaje.

\subsubsection{Garantías estatales para la educación superior de DV}

Las garantías que el Estado puede otorgar sobre calidad de la educación e igualdad de condiciones para los alumnos con DV es un tema que los preocupa y, agregan, como a todos quienes están en las instituciones de educación superior en el país. Ni en nuestro país ni en los países desarrollados estaría asegurado el acceso a una educación de calidad para todos, y ellos estarían en una desventaja mayor por su carácter de minoría.

Los jóvenes entrevistados ingresaron a la universidad a través de un mecanismo que otorga cupos especiales para alumnos discapacitados. Este mecanismo implica que no tienen que rendir la prueba de ingreso común de las universidades tradicionales (PSU), aunque cabe destacar, sí se sentían capacitados para hacerlo. Sin embargo, este tipo de ingreso requiere que las personas con DV cumplan un conjunto de exigencias fijadas por cada universidad, entre las que se incluyen exigencias particulares tales como documentar su rendimiento escolar en la enseñanza media, realizar pruebas de conocimientos específicos, de asignaturas o en áreas relevantes en una carrera, entrevistas personales con profesores y jefes de carrera, y manejo de aspectos instrumentales específicos como uso del bastón en forma independiente, tener un sistema de lectura y escritura efectivo (Braille y ayudas ópticas y no ópticas para aquellos con baja visión), y uso de tecnología computacional en el nivel de usuario. En relación con las adecuaciones curriculares que requieren, coinciden en señalar que acuerdan directamente con sus profesores la forma en que serán evaluados, por ejemplo, si la evaluación será oral o escrita.

\subsubsection{Instancias de apoyo especializado en las universidades, exigencias académicas e inciden- cia de la DV en el rendimiento}

En el país, algunas universidades han incorporado gradualmente instancias de apoyo específicas para personas con diversos tipos de discapacidad. Los jóvenes perciben que estos recursos han ido incrementándose y que los alumnos con discapacidad cuentan con beneficios que facilitan su acceso a contenidos y materias, y valoran positivamente las ayudas concretas para abordar los contenidos de las asignaturas. Opinan que estas ayudas nunca son suficientes o totalmente efectivas para los requerimientos de un estudiante con DV, no obstante, consideran que han significado un gran avance en la educación de y para personas con discapacidad.

Por otra parte, el rendimiento de un alumno con DV en la universidad sería equivalente al rendimiento de un alumno promedio, según lo señalado por ellos. Algunos serían más aventajados mientras otros presentarían algunas dificultades. El rendimiento dependería tanto de la carrera escogida como de sus propias habilidades cognitivas y sociales y capacidad de organización del tiempo.

Los jóvenes dan cuenta también de situaciones inesperadas durante el proceso formativo, como cuestionar que un alumno pueda realizar una asignatura poniendo como limitante su condición visual, y que indicarían dificultades de planificación y coordinación en las carreras que promueven el ingreso de estudiantes con DV. Aceptar a un alumno 
con DV implica el análisis y planeamiento de la malla curricular, y de las adecuaciones necesarias que pudiera requerir, lo que al parecer no siempre sucede.

\subsubsection{Valor de la independencia y relaciones sociales en la universidad}

Los jóvenes perciben que desde su ingreso a la universidad han tenido un recorrido complejo y han vivido muchas situaciones, a veces con resultados exitosos. Entre otras, han tenido que resolver cómo desplazarse con su bastón por lugares desconocidos y con una distribución a la cual no están acostumbrados, y han debido entablar conversación con desconocidos para obtener información indispensable o por la necesidad de acceder a ciertos lugares. Todavía les cuesta enfrentar algunas situaciones, pero expresan que están dispuestos a continuar haciendo esfuerzos por mejorar. Al mismo tiempo perciben que la posibilidad de encontrarse en nuevos contextos sociales les ha permitido abrirse a otros espacios físicos y sociales, y que sus características personales y la mantención de actitudes positivas y proactivas frente a la vida han sido claves en este aspecto.

La percepción positiva que tienen acerca de sí mismos y de sus logros como personas con discapacidad tiene un alto significado en esta etapa. Está relacionada con haber alcanzado una cierta estabilidad emocional construida con base en diversas experiencias resueltas exitosamente o del aprendizaje de sus fracasos. Esta percepción formaría parte de los recursos que poseen para enfrentar exitosamente la etapa universitaria.

Ellos coinciden en que ésta es una etapa distinta y complicada, están más solos y lo sienten así. La universidad es una instancia más competitiva, donde todos están en una carrera por superarse y ser mejores, y eso resta tiempo para hacer nuevas amistades o darse espacios con otros. Tienen amigos, pero dicen que sólo permanecen las relaciones más cercanas y verdaderas. Además, consideran que en la universidad quedan al descubierto carencias de la mayoría de los jóvenes y de la sociedad en su conjunto, tales como dificultades para convivir con la diversidad, y que se hace más evidente la falta de empatía y solidaridad, y perciben que en el ambiente prevalece el individualismo. También se han hecho más conscientes de sus propias debilidades.

\subsubsection{La relación con los profesores en la universidad}

Los jóvenes concuerdan en que el trato de los profesores es distinto en la universidad, en comparación con la experiencia que tuvieron durante la educación básica y media. Perciben una relación más abierta, más flexible y autónoma, pero también más exigente, y donde la balanza se inclina en función de lo que puedan hacer o demostrar a través de sus logros. También destacan y valoran positivamente que frente a las exigencias el trato de los profesores es igualitario, sienten que son exigidos al igual que los demás estudiantes.

En sus relatos también surge la presencia de algunos profesores con mala disposición y que ponen barreras para trabajar en sus clases con alumnos con DV, pero parecieran constituir una minoría; además, sienten que ahora manejan algunas estrategias para revertir una situación de este tipo o buscar alternativas. En general, la experiencia adquirida en el pasado influye porque les permite contar con más habilidades para relacionarse con sus profesores: ahora son ellos quienes presentan sus inquietudes o dificultades, y creen que depende de sí mismos llegar a acuerdos que los favorezcan. Sienten que conocen las respuestas para múltiples situaciones y que, de alguna manera, gestionan su propio quehacer educativo. 


\subsection{ANÁLISIS CRÍTICO GLOBAL DEL PROCESO DE IE DESDE LA PERSPECTIVA DE LOS JÓVENES}

Cada joven participante en este estudio hace un análisis del proceso de IE desde su propia perspectiva, y con énfasis diferentes. En general, su análisis global del proceso contiene aspectos positivos y negativos e incluye las responsabilidades de todos los involucrados en él, incluidos ellos mismos. Así, en relación con el rol de sus padres se centran en los estilos de crianza y cómo estos incidieron en su proyección personal. Sobre la institución de apoyo, analizan sus propias individualidades dentro de un grupo con necesidades diversas. En cuanto al establecimiento educativo común, hacen énfasis en los diferentes niveles de compromiso existentes en su interior. Y respecto de sí mismos, se refieren como influidos y moldeados por todo lo anterior, pero con características que los hicieron capaces de salir adelante, concluir la educación básica y media y llegar a la universidad para optar a una profesión. Destacan que el camino recorrido no ha sido fácil y que el apoyo de la familia, amigos e instituciones que los han acompañado les ha facilitado llegar a donde se encuentran actualmente.

Por otra parte, se sitúan como observadores para analizar la IE como fenómeno social, intentando ir más allá de sus propias experiencias. Algunos se refieren al proceso de integración a partir de las contradicciones que perciben entre las políticas sociales y educativas, y la situación respecto de la IE en la sociedad como un todo. Específicamente, perciben que esas políticas promueven situaciones que a su parecer sólo son posibles en el papel, mientras que la sociedad no las asume y procesa los cambios necesarios con mucha lentitud. Señalan que desde sus primeros años de escuela hasta ahora, la percepción que nuestra sociedad tiene acerca de los discapacitados visuales ha avanzado mucho, pero que todavía hay bastante camino que recorrer. También plantean que es en las escuelas o liceos donde en general se requieren las mayores transformaciones. Además, cuando se refieren a las experiencias vividas a través de los años, son conscientes del efecto directo del trato recibido en los establecimientos educativos sobre su forma actual de enfrentar la vida.

Mientras unos enfatizan lo difícil que ha sido vivir con una discapacidad por la discriminación experimentada, y cómo ésta en algún momento marcó negativamente su autoimagen y autoestima, otros señalan que el impacto no fue tan fuerte, sobre todo, porque reconocen en su ambiente inmediato un rol de contención y apoyo presente a lo largo de todo el proceso de IE y de su vida en general. En términos globales son críticos de aquellos pares con DV que están resentidos y buscan generar sentimientos de autocompasión en otros, como también de algunas personas con DV que se autovictimizan. Creen que dan una imagen sesgada que no corresponde a la mayoría de las personas con DV, y que de alguna manera las daña a todas.

En sus relatos, aparecen satisfechos con los logros que han alcanzado y afirman que si bien las cosas no han sido fáciles, han llegado donde querían estar o donde alguna vez pensaron llegar. También es interesante que algunos atribuyen sus logros principalmente a capacidades personales y secundariamente a motivos externos, en cambio, otros los atribuyen al rol de su entorno social inmediato que sería clave como facilitador del proceso vivido y les habría permitido llegar donde están. Además, un dato relevante que se repite es que son los únicos de sus hermanos que lograron ingresar y terminar una carrera en la educación superior. Ellos se perciben a sí mismos como personas normales, con características propias de su edad y, aunque la ceguera o DV sería una condición que los hace distintos en ciertos aspectos, se conciben básicamente parte del todo que es la sociedad. 


\section{DISCUSIÓN Y CONCLUSIONES}

Este estudio comenzó interrogándose sobre cómo jóvenes estudiantes universitarios discapacitados visuales perciben su experiencia de integración en el sistema educativo $\mathrm{y}$, específicamente, qué aspectos de este proceso perciben como facilitadores $\mathrm{u}$ obstaculizadores. Un supuesto enunciado en el título del estudio es que los cinco jóvenes participantes habían logrado hacer el proceso de integración educativa en forma exitosa, siendo su condición de estudiantes universitarios una evidencia de los logros en este proceso. Los resultados obtenidos permiten sostener que este supuesto era correcto.

Estos jóvenes son protagonistas del proceso de cambios iniciado en el país en la década de los 90', que pretendió lograr la integración educativa de los niños con NEE en nuestro sistema educativo, y que forma parte de un movimiento mundial que puso en el centro la igualdad de derechos de las personas con discapacidad y el derecho de los niños discapacitados a educarse junto con los demás niños en las escuelas comunes, esto es, donde las escuelas son concebidas como un espacio de construcción democrática en que se expresa el derecho humano de la igualdad (Hevia, 2010), y la educación se constituye en un bien básico común para todos (Bueno Aguilar, 2008). Desde la perspectiva de los jóvenes participantes, este proceso en curso tiene algunas luces pero todavía tiene muchas sombras.

A partir de su experiencia, pareciera que el marco de principios normativos y éticos, tanto en términos sociales amplios como en términos pedagógicos en un sentido más estrecho, no ha sido suficiente todavía para producir cambios profundos. Primero porque no ha logrado que los cambios penetren en las creencias enraizadas en nuestra sociedad hacia las personas con discapacidad, ni ha eliminado las prácticas discriminatorias dirigidas hacia ellos (Tuttle $\&$ Tuttle, 2004). Segundo, porque tampoco ha influido lo suficiente en las creencias y la acción pedagógica del conjunto de los profesores de los establecimientos comunes que tienen proyectos de integración educativa. Esta percepción coincide con lo planteado por Arias et al. (2005), a saber, que la puesta en marcha del proceso de IE no encontró una sociedad ni una comunidad educativa bien preparadas, y que al sistema educativo le faltaban las condiciones necesarias para realizar el ideal que suponía, y también con los planteamientos de la Comisión de Expertos de Educación Especial (MINEDUC, 2004), que apuntó a la inconsistencia entre el diseño de las políticas y su implementación en el país.

Los jóvenes señalaron experiencias de discriminación entre pares mal manejadas por los profesores y directivos de los establecimientos comunes. En especial, enfatizaron experiencias altamente negativas especialmente en la educación básica, con un número muy importante de profesores sin preparación adecuada, y que al parecer veían la presencia de estudiantes con DV en sus aulas como una imposición, otras veces, mostrando prejuicios habituales sobre ellos en nuestra sociedad y, lo que es más grave todavía, siendo excluyentes mediante conductas de indiferencia o simplemente ignorando su presencia en la sala de clases, sin compromiso e iniciativa para resolver en la práctica pedagógica los desafíos de adecuación curricular necesarios para sus NEE, y porque no percibieron que hubiera una búsqueda de asistencia técnica en los profesores de los centros de apoyo cercanos. Perciben que esos problemas estaban más presentes en los profesores de mayor edad, en quienes habría más resistencias al cambio y, en general, atribuyeron esas actitudes y comportamientos al miedo, a lo amenazante que les resultaba no ser capaces de manejar apropiadamente un aula integrada con un niño o joven con DV, y que no sabían cómo enfrentar o qué hacer en términos de su acción pedagógica. 
Está demás señalar que estas experiencias resultaron difíciles y muy dolorosas -hasta traumáticas- para los jóvenes, y que sin la presencia de otros factores que los protegieron, a los que se hará referencia más adelante, podrían haber afectado su proceso de IE de manera tan severa que incluso implican el riesgo de abandono del sistema educativo o resultados académicos deficientes o apenas regulares. Más allá de cuáles fueran las razones presentes en estos profesores de los establecimientos comunes, si ellos eran pocos o muchos, los hechos indican fallas en la implementación de la IE que podrían ser resueltas parcialmente con algunas medidas que provean a los profesores de herramientas básicas, pero necesarias y suficientes en calidad y cantidad, para que puedan enfrentar mejor el proceso de IE. El informe de la Comisión de Expertos de Educación Especial (MINEDUC, 2004) sugirió que los obstáculos comienzan en la formación inicial de los profesores de la escuela regular, porque no incluye una instrucción que les permita atender las NEE de los alumnos, y que lo mismo sucede durante su formación en servicio. Dicho informe alerta que esta situación también se replica en la formación inicial de otros profesionales de la educación regular y especial, a lo que se añade que la formación de profesores especialistas aún mantiene un enfoque clínico (MINEDUC, 2004), tal como lo confirman los estudios de Vega (2009), Damm (2009) y Tenorio (2011). Los profesores han sido preparados para atender la diversidad desde el currículo común y no saben cómo adaptarlo ni diversificarlo (Tenorio, 2005), requiriendo algunas capacidades mínimas -que no tienen- para trabajar en IE en el aula (Consultora en Estudios, Asesorías y Planificación en Desarrollo Local, 2004). Resulta necesario un esfuerzo global del sistema educativo para reforzar las orientaciones pedagógicas y normativas y capacitar a los profesores comunes y especiales en ejercicio, de manera que el temor y las resistencias cedan paso a una práctica pedagógica más comprometida con los valores de la igualdad y el respeto a la diversidad, esfuerzo que sería más efectivo si comenzara a hacerse durante la formación inicial de los profesores.

Por otra parte, la situación descrita por los jóvenes también estaría mostrando que los PIE no forman una parte efectiva de los proyectos educativos de las escuelas comunes, y que es muy posible que estos proyectos sean vistos como una actividad paralela, o un asunto de algunos profesores o, incluso, que implica solamente a aquellos que tienen niños integrados en sus aulas, como lo plantea Marchesi (2001). De aquí deriva también una sugerencia dirigida a que la formación de directivos incorpore este tipo de contenidos y principios educativos durante su formación como tales, y en las materias que tienen que ver con la gestión de la discapacidad en sus establecimientos y la creación de redes de apoyo.

En relación con los profesores de apoyo, Arias et al. (2005) señalaron la necesidad de lograr que los profesores especialistas y los profesores de aula común trabajen en conjunto, para que los últimos tengan seguridad e información que permita resolver sus inquietudes, y que los primeros deben orientar a la comunidad educativa y a los padres de los niños con NEE. La asesoría y el apoyo implican una co-construcción conjunta de criterios y acuerdos con el profesor común, que pueden influir en el trabajo con los padres y en aspectos pedagógicos tales como compartir metodologías, experiencias, formas de relacionarse, enfrentar temores, entre otros, en que ambos pueden enriquecerse (Blanco, 1999). Asimismo, lo anterior tiene que ver con el desarrollo de comunidades educativas en las cuales los padres de los estudiantes con DV estén efectivamente integrados, tarea que debería ser del/la director/a del establecimiento común. Es necesario replantearse la manera en que se involucran los padres con la escuela, asegurarse que tengan información y comprendan los objetivos de aprendizaje, y también que conozcan los logros educativos de sus hijos (Centro de Estudios POLITEIA y MINEDUC, 2008). 
Respecto al proceso de IE en sí mismo y, específicamente, las experiencias de los jóvenes con los obstaculizadores y facilitadores del proceso, los resultados muestran que el proceso es vivido como un continuo, sin lagunas o interrupciones pero, sobre todo, más como un proceso de inclusión social que solamente de integración al sistema educativo. Éste ha sido el eje de sus vidas, y sus principales preocupaciones, problemas y reflexiones han girado en torno a dicho proceso. Sus comentarios sobre el tiempo que dedicaron durante toda la etapa escolar a su doble jornada educativa, en el establecimiento educativo común y en el centro de apoyo, y la crítica a algunos aspectos del estilo de crianza de sus padres, que no les permitió o no les dio el suficiente tiempo para desarrollar otras actividades frecuentes entre los niños y jóvenes de la misma edad, dan cuenta de ello. En la etapa universitaria, los jóvenes vuelven a girar en torno a sus desafíos y en cómo enfrentar las demandas de sus estudios, aunque destaca que algunos refieren la mantención de relaciones sociales con amistades que al parecer hicieron anteriormente. Por otra parte, la educación aparece con una gran importancia en sus relatos, y en esa valoración sus padres jugaron un papel muy trascendente. Queda suficientemente establecido que sus padres les transmitieron que la educación era lo más importante para sus vidas futuras.

En el proceso de integración educativa se ven a sí mismos enfrentando y resolviendo problemas que les permiten ir adquiriendo experiencias y les proporcionan aprendizajes, que ahora están aprovechando en su periodo universitario, por ejemplo, siendo menos pasivos para relacionarse con sus profesores y aplicando estrategias más efectivas aprendidas anteriormente. También perciben que desarrollaron diversas capacidades que les sirven de herramientas a través de la vida y, en general, se perciben exitosos. Sienten que han logrado vencer muchas barreras, lo cual contribuye a que posean una buena autoestima y se sientan autoeficaces respecto de sus metas, por ejemplo, para terminar sus carreras. Ellos destacan que son los únicos entre sus hermanos que han logrado llegar al nivel de educación superior, y el sólo hecho de haber llegado a este nivel es una demostración de sus capacidades.

Otra característica del proceso, especialmente clara a partir de la pubertad y la adolescencia, es que viven las etapas del ciclo vital como lo haría cualquier otro niño o adolescente. Esto se observa en el proceso de búsqueda de identidad y en los problemas de autoestima asociados, en la preocupación por su apariencia física, o en sus dificultades para hacer amistades, entre otros, proceso que siempre aparece marcado y a veces re-marcado por su condición de discapacidad visual. Así, los contenidos específicos de dicho proceso aluden la mayoría de las veces a su discapacidad y recuerdan lo que plantean Tuttle \& Tuttle (2004) en cuanto a que los mismos principios establecidos en la psicología general serían adecuados para esta condición, pero también que su diferencia respecto de otras personas tiene que ver principalmente con su experiencia y con las limitaciones comportamentales que son propias de su discapacidad (Cit. en Tuttle \& Tuttle, 2004: 37).

El proceso de IE es complejo y difícil, y en él los jóvenes están expuestos a vivir muchas veces experiencias dolorosas asociadas con discriminación y menoscabo por su condición, sin embargo, les molestan las expresiones de lástima, que los hacen sentirse humillados o tratados indignamente, tanto como las conductas de otras personas con discapacidad visual que perciben como resentidas por ese trato o que se victimizan. Estos jóvenes poseen capacidad introspectiva y son conscientes de los factores que más influyeron y siguen influyendo en su proceso de IE, y en general en sus vidas, y son capaces de evaluarse con sus debilidades y fortalezas. Además, parecen haber reflexionado en forma previa a las entrevistas sobre este proceso. Así, los jóvenes perciben con mucha 
claridad cuáles son los factores que obstaculizaron su proceso de IE y cuáles lo facilitaron de manera fundamental. Estos son factores externos de su entorno familiar y educativo más inmediato que, desde la perspectiva de los jóvenes participantes, tuvieron una presencia muy importante desde los inicios del proceso de integración hasta llegar al nivel de estudios universitarios. Constituyen factores del proceso que actúan de manera permanente, y cuya presencia, ausencia y la forma en que se manifiestan los afectaron fuertemente a través del tiempo, ya sea con un impacto positivo o negativo que persiste y es evidente. De este modo, dependiendo de las circunstancias y forma en que se manifiestan, un factor puede actuar en ocasiones como facilitador y en otras como obstaculizador del proceso de integración.

Los factores externos que priman como facilitadores del proceso de IE son los padres, otras personas significativas, las instituciones de apoyo y algunos aspectos técnicos vinculados con los procesos de enseñanza-aprendizaje. Los tres primeros factores, o más bien agentes del proceso de IE, juegan también un rol de protectores de la salud mental y bienestar psicológico de los jóvenes, especialmente, cuando ellos han vivido experiencias de carácter discriminatorio. Los factores externos que se manifiestan principalmente como obstaculizadores o barreras para la integración son las relaciones con sus pares y profesores en los establecimientos de educación común básica y media, no así en la etapa de estudios universitarios.

Otro factor externo que facilita el proceso de IE en los establecimientos comunes está constituido por aspectos técnicos referidos a diversas adecuaciones curriculares que contribuyen a desarrollar diferentes habilidades en los niños y jóvenes con DV, incluyendo el uso de recursos tecnológicos apropiados para ellos. Llama la atención que este factor aparece vinculado especialmente con intervenciones impulsadas desde el centro de apoyo hacia los establecimientos educativos comunes, o que éste realiza en forma paralela a las actividades de los establecimientos, y que no sean acciones pedagógicas nacidas en el seno de los propios establecimientos educativos comunes. En la universidad perciben presencia de estos recursos e instancias de apoyo, que habrían ido creciendo pero no serían suficientes todavía. Al mismo tiempo, consideran que los problemas de calidad de la educación en el país también los afectan, pero que como minoría tienen más desventajas al respecto.

Por otra parte, durante el proceso de IE también surgen en los jóvenes recursos personales internos, de tipo psicológico, principalmente la capacidad de actuar con autonomía e independencia, y que son favorecidos por los factores externos facilitadores presentes. Ellos perciben que logran gradualmente ciertos niveles de independencia que les permitirían tener sentimientos de autonomía y autodeterminación sobre sus vidas, que elevan su autoestima y autoeficacia, y aumentan sus posibilidades de ser autovalentes. También surgen recursos relacionales, principalmente habilidades sociales que van desarrollando y fortaleciendo a través del proceso de IE, y que estiman indispensables para una integración educativa efectiva y exitosa, pero, sobre todo, para su inclusión social.

Sobre el desarrollo de sus capacidades para el proceso de IE y para sus vidas evalúan que los centros de apoyo y sus padres enfatizaron el logro de habilidades cognitivas, posiblemente porque las demandas y exigencias académicas eran más importantes para ellos, mientras que las habilidades sociales, que serían esenciales para la vida, quedaron en un segundo plano, manifestándose ahora que son estudiantes universitarios como déficits. Al respecto, también expresan que estas dificultades para la vida social podrían afectar negativamente su vida laboral futura. Además, la enseñanza de las técnicas de orientación y movilidad y de las actividades para la vida diaria tuvo menos importancia en su formación que la que ellos le conceden actualmente, por su rol en el logro de la independencia y la autonomía social y psicológica. 
Lo anterior sugiere, tanto para los centros de apoyo como para la orientación a los padres, la búsqueda de un mejor equilibrio en el desarrollo de las habilidades. También incluye que los equipos de apoyo cuenten con herramientas para guiar a los padres y a los profesores de los establecimientos comunes en el manejo de sus ansiedades y temores. De sus relatos se desprende que ellos se sienten favorecidos por la fuerte alianza entre sus padres y el centro de apoyo (COALIVI) para su proceso de IE, y de inclusión social. Sus opiniones sugieren que ambos estuvieron comprometidos fuertemente con su integración en los establecimientos comunes, y que sus padres actuaron con convicción asumiendo grandes riesgos en estrecha colaboración y siempre soportados por los profesionales del centro de apoyo.

Por último, cabe destacar que las limitaciones más importantes de este trabajo tienen que ver con las características del diseño del estudio: un estudio de casos. A partir de los resultados de esta indagación, se sugiere que a futuro otras investigaciones se centren en las diferencias de género que no fueron exploradas, en jóvenes que no tuvieron éxito en su integración, en los padres, en los directivos y en los profesores de los establecimientos comunes, y en los profesionales de los centros de apoyo. Queda mucho por conocer y aprender desde la perspectiva de todos ellos.

\section{REFERENCIAS BIBLIOGRÁFICAS}

Ainscow, M. (2001). Desarrollo de escuelas inclusivas. Ideas, propuestas y experiencias para mejorar las instituciones escolares. Madrid: Narea.

Arias, I., Arriagada, C., Gavia, P., Lillo, L. y Yáñez, N. (2005). Visión de la integración de niños/as con Necesidades Educativas Especiales. Tesis de Pregrado, Departamento de Educación, Universidad de Chile. Recuperado de http://www.tesis.uchile.cl/tesis/uchile/2005/arias_i/sources/ arias_i.pdf, consultado en abril de 2011.

Bermejo, M. L., Fajardo, M. I. y Mellado, V. (2002). El aprendizaje de las ciencias en niños ciegos y deficientes visuales. Integración. Revista sobre Ceguera y Deficiencia Visual, n. 38, 25-34. Recuperado de http://www.once.es/educación, consultado en abril de 2011.

Blanco, R. (2006). La equidad y la inclusión social: Uno de los desafíos de la educación y la escuela de hoy. Revista Electrónica Iberoamericana sobre Calidad, Eficacia y Cambio en Educación, vol. 4, n. 3, 1-15. Recuperado de http://www.rinace.net/arts/vol4num3/art1.pdf, consultado en marzo de 2012. (1999). Hacia una escuela para todos y con todos. Boletín Proyecto Principal de Educación en América Latina y el Caribe $N^{\circ} 48$ (abril). Santiago de Chile: UNESCO/OREALC.

Bueno Aguilar, J. (2008). Nuevos retos, nuevas perspectivas para la Educación Multicultural. Revista Educación Inclusiva, n. 1, 59-76. Recuperado de http://dialnet.unirioja.es/descarga/ articulo/3011825.pdf, consultado en marzo de 2012.

Cámara de Diputados. Departamento de Evaluación de la Ley. (2012). Evaluación de la Ley 20.422 (noviembre). Recuperado de http://www.evaluaciondelaley.cl, consultado en abril de 2013.

Centro de Estudios POLITEIA y MINEDUC. (2008). Estudio mejoramiento de la gestión y la calidad de la educación municipal. Santiago de Chile. Recuperado de http://www.mineduc.cl, consultado en junio de 2011.

CLADE, CEJIL y Muñoz, V. (2009). Derecho a la educación de las personas con discapacidad en América Latina y el Caribe. Informe para la Comisión Interamericana de Derechos Humanos (noviembre). Recuperado de http://cejil.org/sites/default/files/derecho_educacion_personas_ discapacidad_ALyCaribe.pdf, consultado en abril de 2013.

Consultora en Estudios, Asesorías y Planificación en Desarrollo Local (CEAS). (2003). Estudio a nivel muestral de la calidad del proceso de integración educativa. Santiago de Chile. Recuperado 
de http://www.mineduc.cl, consultado en enero de 2012.

Crosso, C. (2010). El derecho a la educación de personas con discapacidad: Impulsando el concepto de Educación Inclusiva. Revista Latinoamericana de Educación Inclusiva, vol. 4, n. 2, 79 95. Recuperado de http://www.rinace.net/rlei/numeros/vol4-num2.html, consultado en abril de 2013.

Damm, X. (2009). Representaciones y actitudes del profesorado frente a la integración de niños/ as con Necesidades Educativas Especiales al aula común. Revista Latinoamericana de Educación Inclusiva, vol. 3, n. 1, 25-35. Recuperado de http://www.rinace.net/rlei/numeros/vol3-num1/art2.pdf, consultado en enero de 2013.

Duero, D. y Limón, G. (2007). Relato autobiográfico e identidad personal: Un modelo de análisis narrativo. Revista de Antropología Iberoamericana, vol. 2, n, 2. 232-275. Recuperado de http://www. aibr.org/antropologia/02v02/articulos/020202.php, consultado en octubre de 2011.

Fundación HINENI. (2002). Servicios de apoyo, principios y orientaciones. Santiago de Chile. Recuperado de http://www.mineduc.cl, consultado en abril de 2011.

Gairín, J. (Coord.). (2012). La organización y atención a la diversidad en centros de atención secundaria de Iberoamérica. Reflexiones y experiencias. Santiago de Chile: Red AGE.

Gil, J. (1994). Análisis de datos cualitativos. Aplicaciones a la investigación educativa. Barcelona: PPU.

Hevia, R. (2010). El derecho a la educación y la educación en derechos humanos en el contexto internacional. Revista Latinoamericana de Educación Inclusiva, vol. 4, n. 2, 25-39. Recuperado de http://www.rinace.net/rlei/numeros/vol4-num2/art1.pdf, consultado en abril de 2013.

Marchesi, A. (2001). Cambios sociales y cambios educativos en Latinoamérica. Madrid: Universidad Complutense de Madrid.

MINEDUC. (2004). Nueva perspectiva y visión de la educación especial. Comisión de Expertos de Educación Especial. Santiago de Chile. Recuperado de http://www.mineduc.cl, consultado en abril de 2011.

(1990a). Decreto 490, Ley 19.284 de integración de las personas con discapacidad. Santiago de Chile. Recuperado de http://www.mineduc.cl, consultado en abril de 2011.

(1990b). Planes y programas de estudio para educandos con déficit visual. Exento $N^{o} 89$.

Santiago de Chile. Recuperado de http://www.mineduc.cl, consultado en abril de 2011.

Sierra, F. (1998). Función y sentido de la entrevista cualitativa en investigación social. En J. Galindo (Coord.), Técnicas de investigación en sociedad, cultura y comunicación (pp. 277-354). México D.F.: Pearson.

Tenorio, S. (2011). Formación inicial docente y necesidades educativas especiales. Estudios Pedagógicos, vol.XXXVII, n. 2,249-265. Recuperado de http://www.scielo.cl/scielo.php?pid=S071807052011000200015\&script=sci_arttext, consultado en marzo de 2012.

(2005). La integración escolar en Chile: Perspectiva de los docentes sobre su implementación. Revista Electrónica Iberoamericana sobre Calidad, Eficacia y Cambio en Educación, vol. 3, n. 1, 823-831. Recuperado de http://www.ice.deusto.es/RINACE/reice/Vol3n1_e/ Res_Tenorio.htm, consultado en abril de 2011.

. y González, G. (2004). Integración escolar y efectividad en la escuela regular chilena. Revista Digital Umbral, n. 16, 1-14. Recuperado de http://www.galeon.com/documentosmc/ integracion.pdf, consultado en abril de 2011.

Tuttle, D. \& Tuttle, N. (2004). Self-Esteem and Adjusting with Blindness. The Process of Responding to Life's Demands. Illinois: Charles C. Thomas Publishers.

Valcarce, M. (2011). De la escuela integradora a la escuela inclusiva. Innovación Educativa, n. 21, 119-131. Recuperado de http://www.usc.es/revistas/index.php/ie/issue/view/4, consultado en abril de 2013.

Vega, A. (2009). Integración de alumnos con necesidades educativas especiales: ¿Existe coherencia entre el discurso y las prácticas pedagógicas ejercidas por los profesores básicos? Estudios Pedagógicos, vol. XXXV, n. 2, 189-202. Recuperado de http://www.scielo.cl/scielo.php?pid=S071807052009000200011\&script=sci_arttext, consultado en marzo de 2012. 